\title{
Length of Left behind Time, Psychological Capital and Cell Phone Dependence of College Students
}

\author{
Xiangna Kong ${ }^{1,2}$, Chang Liu $^{3}$ \\ ${ }^{1}$ Education College of Hubei University of science and technology, Xianning, Hubei, China \\ ${ }^{2}$ Key Research Base of Humanities and Social Sciences in Hubei Province, Rural education and \\ Cultural Development Research Center, Xianning, Hubei, China \\ ${ }^{3}$ Wuhan sunshine Social Work Service Center, Wuhan, China
}

Keywords: Length of Left behind Time; Psychological Capital; Cell Phone Addiction; Intermediary Role.

Abstract: Objective: To explore the relationship among the length of left behind time, psychological capital and cell phone dependence of college students. Methods: 276 students were randomly selected from a university, the length of left behind time of them was investigated, meanwhile Positive Psychological Capital Questionnaire(PPQ )and Smartphone Addiction Scale of College Students (sas-c) were used to measure them. Results:(1) The level of psychological capital of students with left behind experience was significantly lower than that of students without left behind experience, and the degree of mobile phone addiction of college students with left behind experience was significantly higher than that of college students without left behind experience;(2) There was a significant negative correlation between the length of left behind and psychological capital, and there was a significant positive correlation between the length of left behind and mobile addiction, meanwhile there was a significant negative correlation between psychological capital and cell phone addiction.(3) Psychological capital played a significant intermediary effect between mobile phone addiction and staying time. Conclusion: We can prevent left behind children's cell phone addiction by increasing their psychological capital.

\section{Introduction}

China has a large number of left behind children. One or both of their parents go out to work, leaving them to live in the place where their household registration is located. Their psychological problems are prominent: loneliness, depression, lack of social support. They communicate with parents from other places through mobile phones and Internet, and at the same time, they are easy to sink into the mire of Internet. Some studies had found that junior high school students were seriously addicted to mobile phones,and left behind students are more addicted to mobile phones than non left behind students [1].When they grow up and enter university, do these problems still exist? Psychological capital is a kind of positive psychological state in the process of individual growth and development. Fan Sihua and Yu Si found that left behind children's psychological capital and well-being were lower [2]. Zhang Guojin and Yao Zhiqiang found that there was a significant negative correlation between the positive psychological capital of college students and 
mobile phone dependence [3]. So, what will be the relationship between College Students' length of left behind time and their psychological capital, cell phone addiction?

\section{Method}

Randomly select a college student as the research object. 300 questionnaires were sent out, 276 were recovered, 248 were valid. Among them, there were 136 boys and 112 girls, 47 freshmen and 62 sophomores, 67 students in grade three and 72 in grade four.

\subsection{Research tools}

(1)Length of left behind time questionnaire

This part was to investigate :When college students were growing up ( $\geq 16$ years old), how much time did they had to be left behind in their hometown because both parents or one of them left their hometown and went to work in other palce.

(2) Positive Psychological Capital Scale(PPQ)

Positive Psychological Capital Questionnaire compiled by Zhang Kuo et al was used. [4] It has 26 items, including 4 dimensions: self-efficacy, resilience, hope and optimism. It uses Richter's 7 -grade score, from complete inconformity to full compliance. The higher the score, the stronger the psychological capital. The Cronbach alpha coefficient of the questionnaire is 0.90 , and the structure validity of the questionnaire is good, and the item differentiation is above 0.6 , which has good reliability and validity index.

(2) Smartphone Addiction Scale of College Students

Smartphone Addiction Scale of College Students (SAS-C)compiled by Su Shuang et al was used $^{[5]}$, It has 22 items, including 6 dimensions: withdrawal behavior, Salient behavior, social comfort, negative impact, APP use, APP update. The questionnaire uses Likert grade 5 score, from very inconsistent to very consistent, 1-5 points. The higher the score is, the higher the addiction degree of mobile phone will be. The Cronbach alpha coefficient of the total scale is 0.88 , and the retest reliability of the total scale is 0.93 . The scale has good reliability and validity.

\subsection{Statistical Analysis}

Spss22.0 was used to analyze the data, including t-test, correlation analysis, regression analysis and intermediary test.

\section{Results}

\subsection{Differences between Left behind and Non Left behind College Students in Psychological Capital and Cell Phone Addiction}

According to the data we collected, the minimum Length of left behind time of college students in this study was 0 years, that is, there was no stay experience, the longest was 10 years. The group with a left behind time of 0 was named as non left behind group, and the group with left behind experiences was named as left behind group. On the score of psychological capital, the left behind group was significantly lower than the non left behind group. The score of mobile phone addiction in the left behind group was significantly higher than that in the non left behind group. The results were shown in Table 1. 
Table 1. Differences between left behind and non left behind college students in psychological capital and cell phone addiction

\begin{tabular}{lllll}
\hline & $\begin{array}{l}\text { Left-behind } \\
\text { experience }\end{array}$ & M & SD & P \\
\hline psychological & Non-left behind & 156.50 & 10.173 & 0.000 \\
capital & Left behind & 117.56 & 25.012 & \\
Cell-phone & Non-left behind & 37.75 & 9.350 & 0.000 \\
addiction & Left behind & 61.44 & 11.719 & \\
\hline
\end{tabular}

\subsection{The Correlation Analysis of the Length of Left Behind Time, Psychological Capital and} Cell Phone Addiction of College Students

The length of left behind time of college students was negatively related to psychological capital $(\mathrm{r}=-0.752, \mathrm{P}<0.01)$ and positively correlated with cell phone addiction $(\mathrm{r}=0.551, \mathrm{P}<0.01)$, At the same time, their psychological capital was negatively correlated with cell phone addiction $(\mathrm{r}=-0.654, \mathrm{P}<0.01)$. The results were shown in Table2.

Table 2. Correlation coefficient of college students' length of left behind time, psychological capital and cell phone addiction score $(n=248)$

\begin{tabular}{cccc}
\hline Variables & $\begin{array}{c}\text { Length of left behind } \\
\text { time }\end{array}$ & Psychological capital & Cellphone Addiction \\
\hline Length of left behind & $1^{* *}$ & $-0.752^{* *}$ & $0.551^{* *}$ \\
time & & & \\
Psychological capital & $-0.752^{* *}$ & $1^{* *}$ & $-0.654^{* *}$ \\
Self efficacy & $-0.733^{* *}$ & $0.934^{* *}$ & $-0.611^{* *}$ \\
Resilience & $-0.656^{* *}$ & $0.911^{* *}$ & $-0.582^{* *}$ \\
Hope & $-0.674^{* *}$ & $0.955^{* *}$ & $-0.648^{* *}$ \\
Optimism & $-0.735^{* *}$ & $0.933^{* *}$ & $-0.602^{* *}$ \\
Cell phone addiction & $0.551^{* *}$ & $-0.654^{* *}$ & $1^{* *}$ \\
Salient behavior & $0.262^{* *}$ & $-0.343^{* *}$ & $0.637^{* *}$ \\
Withdrawal behavior & $0.503^{* *}$ & $-0.565^{* *}$ & $0.884^{* *}$ \\
Social comfort & $0.596^{* *}$ & $-0.607^{* *}$ & $0.791^{* *}$ \\
Negative impact & $0.341^{* *}$ & $-0.471^{* *}$ & $0.855^{* *}$ \\
APP use & $0.233^{* *}$ & $-0.433^{* *}$ & $0.681^{* *}$ \\
\hline
\end{tabular}

\subsection{Bootstrap Mediation Test}

The bootstrap method was used to test the intermediary effect, and the process program in spss 22.0 was used to test it. Regression analysis results were shown in table 3.It showed that the length of left behind time could significantly predict psychological capital $(\beta=-6.9804, \mathrm{P}<0.05)$, However, the regression coefficient of length of left behind time and psychological capital in predicting mobile phone addiction was not significant. $(\beta=-0.6515, \mathrm{P}>0.05)$, so the sobel test was used. The results were shown in table 4 ,it showed that the intermediary effect was significant $(\mathrm{P}<$ $0.01)$. 
Table 3. Regression analysis

\begin{tabular}{|c|c|c|c|c|c|c|}
\hline $\begin{array}{l}\text { Dependent } \\
\text { variable }\end{array}$ & $\begin{array}{l}\text { Independen } \\
\text { t variable }\end{array}$ & $\mathbf{R}$ & $\mathbf{R}^{2}$ & $\boldsymbol{\beta}$ & $\mathbf{F}$ & $\mathbf{P}$ \\
\hline $\begin{array}{l}\text { Psychologica } \\
\text { I capital }\end{array}$ & $\begin{array}{l}\text { Length of } \\
\text { left behind } \\
\text { time }\end{array}$ & 0.7815 & 0.5648 & -6.9804 & 319.2481 & 0.0000 \\
\hline $\begin{array}{l}\text { Cellphone } \\
\text { addiction }\end{array}$ & $\begin{array}{l}\text { Psychologic } \\
\text { al capital } \\
\text { Length of } \\
\text { left behind } \\
\text { time }\end{array}$ & 0.6603 & 0.4360 & $\begin{array}{l}-0.2847 \\
-0.6515\end{array}$ & 94.6884 & $\begin{array}{l}0.0000 \\
0.0627\end{array}$ \\
\hline
\end{tabular}

Table 4. Sobel test results

\begin{tabular}{cccc}
\hline Effect & se & $\mathrm{z}$ & $\mathrm{p}$ \\
\hline 0.1427 & 0.0205 & 6.9760 & 0.0000 \\
\hline
\end{tabular}

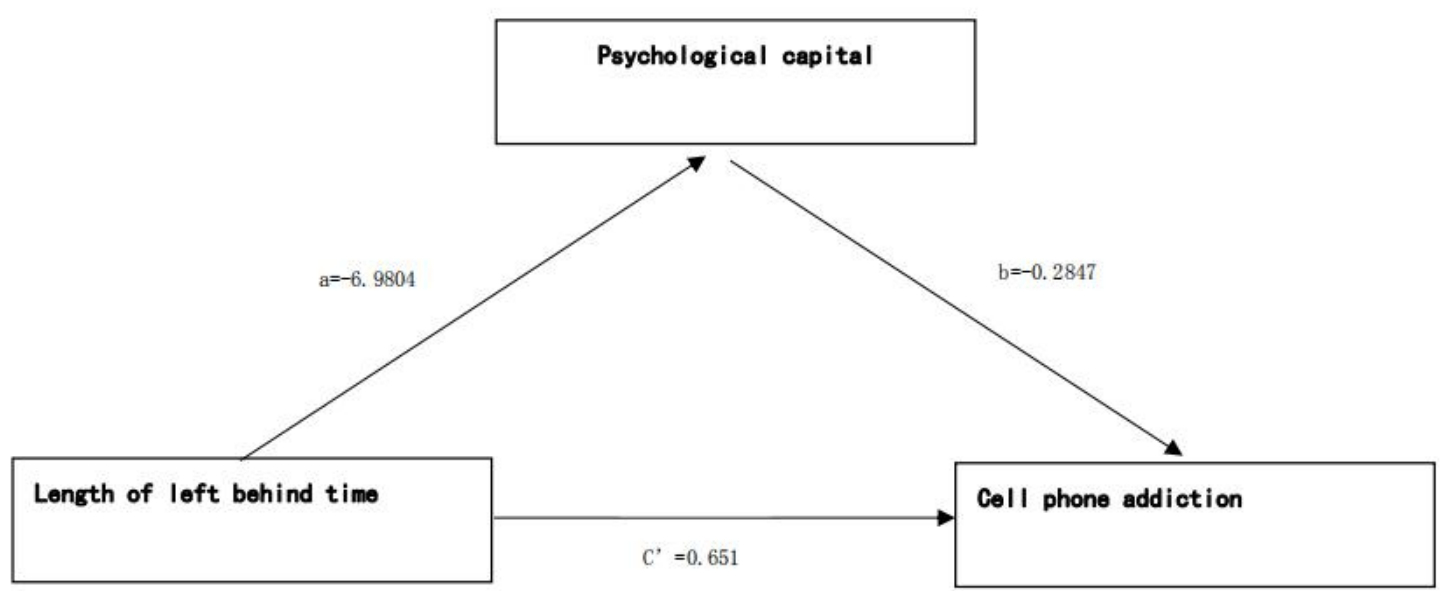

Notes: $A$ is the regression coefficient of left behind time to predict psychological capital; $b$ and c'are the regression coefficients of mental capital and length of left behind time to predict cell phone addiction

Figure 1. The intermediary path of psychological capital in the prediction of cell phone addiction of College Students

\section{Discussions}

The level of psychological capital of college students with left behind experience was significantly lower than that of college students without left behind experience .The results were consistent with those of Xin Tong and Li Hua in 2015 [6]. Yang Xueling's research also found that the psychological resilience of college students with left behind experience was significantly lower than that of college students without left behind experience. Not only that, college students with left behind experience were higher than those without left behind experience in mental illness and suicide risk [7]. These studies have proved that the experience of staying behind was not conducive to the formation of good psychological capital of college students. As a kind of positive psychological state in the process of individual growth and development, psychological capital is 
very important for individual psychology. we also found that the degree of mobile phone addiction of college students with left behind experience was significantly higher than that of college students without left behind experience. In the same way, Xu Jing's research mentioned that because the learning motivation of left behind students was weak, and because their parents were not around, there was no control or lack of control, and they were in an age group with poor resistance to temptation, so they were more likely to be addicted to mobile phones than non left behind students[8].

The following statistics showed that there was a significant negative correlation between the length of left behind time and the total score of psychological capital( $\mathrm{r}=-0.752, \mathrm{P}<0.01)$, and the four dimensions of psychological capital: self-efficacy, resilience, hope and optimism were all negatively correlated. This showed that the longer the left behind time, the lower the psychological capital of college students. This was consistent with the research results of Yang Qin. [9]. There was a significant positive correlation between length of left behind time and cell phone addiction $(\mathrm{r}=0.551, \mathrm{P}<0.01)$. Because parents went out to work, they had no time to care about their children's growth and education, and left behind students lacked control and lived in loneliness, which made them more likely to indulge in the Internet world provided by cell phones, and the longer they left behind, the more likely they were to become addicted to cell phones. At the same time, there was a significant high correlation between the length of left behind time and social comfort in the factors of cell phone addiction $(\mathrm{r}=0.596, \mathrm{P}<0.01)$, This showed that students who had left behind experience had strong social needs, but because of the lack of the ability and opportunity of social interaction in reality, they preferred to use mobile Internet to meet their emotional needs. The negative correlation between psychological capital and cell phone addiction $(\mathrm{r}=-0.654, \mathrm{P}<0.01)$ indicated that the lower the level of psychological capital, the less the ability to deal with difficulties and setbacks, and the more inclined to escape through the virtual mobile phone world, which led to mobile phone addiction. The results of mediating effect showed that psychological capital played a significant mediating role between the length of left behind time and cell phone addiction, and the mediating effect value was 0.43 . The intermediary effect of psychological capital reminds us that for the growing left behind children, the lack of spirit is more unacceptable than the lack of material. In the current environment, even if parents have to go out to work, we can improve and increase the psychological capital of left behind children through various ways, so as to avoid the formation of cell phone addiction in the future.

\section{Conclusion}

(1) The level of psychological capital of students with left behind experience is significantly lower than that of students without left behind experience, and the degree of mobile phone addiction of students with left behind experience is significantly higher than that of students without left behind experience.

(2) The length of left behind time was positively correlated with psychological capital, negatively correlated with mobile phone addiction, and negatively correlated with mobile phone addiction.

(3) Psychological capital played a significant intermediary effect between cell phone addiction and the length of left behind time.

\section{Acknowledgement}

This research was financially supported by the Humanities and Social Sciences Research Project (Department of Education, Hubei Province): "psychological capital research of university with'left behind experience'" (Project ID: 18Y133). 


\section{References}

[1] Yi Xiaoming, Cao Yu. The relationship between Junior middle school students' cell phone addiction and personality [J]. Journal of Neijiang Normal University, 2017,32(12):7-11+53.

[2] Fan Xinghua, She Si, Peng Jia, Fang Xiaoyi. The relationship between Life pressure, loneliness and happiness of left behind children: the mediating and regulating role of psychological capital [J]. Psychological Science,2017,40(02):388-394.

[3] Zhang Guojin, Yao Zhiqiang. The relationship between College Students'positive psychological capital and cell phone dependence [J]. Journal of Gannan Normal University, 2018, 39(02):119-122.

[4] Zhang Kuo, Zhang Jian,Dong Yinghong. Positive mental capital: Measurement and its relationship with mental health [J]. Research on Psychology and Behavior, 2010,8(01):58-64.

[5]Su Shuang,Pan Tingting,Liu Qinxue, Chen Xiaowen, Wang Yujing,Li Mingyue. The development of College Students' smart phone addiction scale [J]. Chinese Journal of mental health,2014,28(05):392-397.

[6]Xing Tong,Li Hua. Research on the psychological capital of the left behind College Students [J]. Journal of Tong ling University,2015,14(04):71-74.

[7]Yang Xueling,Feng Xiangang,Cui Zitian. The left behind experience, psychological resilience and psychopathological symptoms of College Students [J]. Chinese Journal of mental health,2014,28(03):227-233.

[8]XuJing,WangYongbao. The problem of left behind students' addiction to cell phones and educational countermeasures -- from the perspective of "boredom" [J]. Journal of Guangdong Youth Vocational College,2018,32(03):23-27.

[9]Yang Qin,Cai Taisheng,Lin Jing. The influence of left behind experience on College Students' psychological resilience[J].Chinese Journal of health psychology,2014,22(02):272-274. 